\title{
The Thai Version of the Addenbrooke's Cognitive Examination III
}

\author{
Thammanard Charernboon ${ }^{1,2,3 凶}$, Kankamol Jaisin ${ }^{1}$, and Tiraya Lerthattasilp ${ }^{1}$ \\ ${ }^{1}$ Department of Psychiatry, Faculty of Medicine, Thammasat University, Pathumthani, Thailand \\ ${ }^{2}$ Stroke and Neurodegenerative Diseases Research Unit, Thammasat University, Pathumthani, Thailand \\ ${ }^{3}$ Center of Excellence in Applied Epidemiology, Thammasat University, Pathumthani, Thailand
}

The objective of our study was to assess the accuracy of the Thai version of the Addenbrooke's Cognitive Examination III (ACE-T). We used the ACE-T to assess 107 participants aged 60 or over, divided into the following groups: early dementia, $n=30$; mild cognitive impairment (MCI), $n=29$; and normal controls $(\mathrm{NC}), \mathrm{n}=48$. The ACE-T exhibited good internal consistency (0.93) and inter-rater reliability (1.0). The optimal cut-off score for the ACE-T to differentiate MCI from NC was 75/76, giving a sensitivity of 0.9 and specificity of 0.86. At the optimal cut-off of 61/62, the ACE-T had excellent sensitivity (1.0) and specificity (0.97) to distinguish early dementia from non-dementia. The ACE-T had high diagnosis accuracy in the detection of the MCI and early dementia in the Thai population.

Psychiatry Investig 2016;13(5):571-573

Key Words Alzheimer's disease, Cognition, Mild cognitive impairment, Neuropsychological test, Sensitivity and specificity.

\section{INTRODUCTION}

The Addenbrooke's Cognitive Examination (ACE) is a brief cognitive assessment tool that has verified applications in detecting and monitoring cognitive decline in dementia and mild cognitive impairment (MCI). ${ }^{1,2}$

Although the Mini Mental State Examination (MMSE) is likely the most commonly used cognitive assessment tool in Thailand and other countries, several weaknesses have been discussed, including its lack of utility in the detection of MCI and the narrow range of cognitive domains assessed (the MMSE includes no executive function measurements and few assessments of memory and visuospatial ability). ${ }^{3}$ The ACE assesses five cognitive domains: attention/orientation, verbal fluency, language, visuospatial ability, and memory. The original validation study of the ACE showed excellent psychometric properties for diagnoses of both dementia and MCI. While the ACE has been translated for use in many languages worldwide; ${ }^{4-6}$ a Thai version of the ACE has not been previously developed. Moreover, there are only a few validated cognitive

Received: September 12, 2015 Revised: January 5, 2016

Accepted: January 24, 2016 Available online: June 1, 2016

$\triangle$ Correspondence: Thammanard Charernboon, MD, MSc, FRCPsychT Department of Psychiatry, Faculty of Medicine, Phahon Yothin Road, Thammasat University, Pathumthani 12120, Thailand

Tel: +66-2-926-9204, Fax: +66-2-926-9830, E-mail: dr.thammanard@gmail.com

(a) This is an Open Access article distributed under the terms of the Creative Commons Attribution Non-Commercial License (http://creativecommons.org/licenses/by$\mathrm{nc} / 3.0$ ) which permits unrestricted non-commercial use, distribution, and reproduction in any medium, provided the original work is properly cited. assessment tools in Thailand. The objective of this study was to evaluate the validity and reliability of the Thai version of the ACE (ACE-T).

\section{METHODS}

\section{Measures}

The ACE-T, version III, was translated using forward and backward translation methodologies. For forward translation, two psychiatrists familiar with cognitive measurement (TC and $\mathrm{KJ}$ ) translated the original instrument into Thai. Both translators discussed and compared the translations and agreed on a reconciled version. Then, the backward translation was performed by an independent professional English translator who translated the instrument back into English. The final version of ACE-T has minor adaptations from the original version. For instance, naming the American president who was assassinated in the 1960s was replaced with the current monarch of Thailand and the accordion was replaced with the ranat (a Thai musical instrument). Also, instead of identifying the season, participants were asked to tell the time. We then administered the ACE-T to 10 elderly participants as a pilot test. The ACE is scored out of 100 , with a higher score indicating better cognitive performance.

\section{Study design, setting, and population}

The study was carried out at Thammasat University Hospital, located in central Thailand, from January 2014 to June 2015. 
The participants were native speakers of Thai, at least 60 years old, and had at least 4 years of formal education.

We administered the ACE-T to 107 participants: 48 normal controls (NC), 29 patients with MCI, and 30 patients with dementia. People with dementia and MCI were recruited from the Memory Clinic. The clinical diagnoses were established independent of participants' performance on the ACE-T.

Dementia diagnoses were based on the DSM- 5 criteria $^{7}$ for major neurocognitive disorder by expert psychiatrists at the Memory Clinic. To ensure that people with dementia had early dementia, patients with a score of less than 14 on the Thai version of the Mini-Mental State Examination (TMMSE) ${ }^{8}$ were excluded from the study. The diagnosis of MCI was established according to the DSM-5 criteria for minor neurocognitive disorder: ${ }^{7}$ 1) modest decline in cognitive function, 2) modest impairment in cognitive performance [documented by a score of 1.5 standard deviations below the mean normal control score on the Montreal Cognitive Assessment (MoCA $)^{9}$ ], 3) cognitive deficits that do not interfere with independent capacity for everyday activities. The cognitively healthy elderly participants were recruited among relatives or spouses of patients of the Memory Clinic or the general psychiatric clinic. They had no decline in cognitive function, had normal cognitive performance (according the MoCA), and were independent in their daily living activities. Participants with serious neurological, visual/hearing impairment, or psychiatric disorders (e.g., schizophrenia) were excluded.

Patients were evaluated for inter-rater reliability by two investigator psychiatrists (TC, KJ or TL) simultaneously. One investigator administered the ACE-T while another investigator observed and rated the score.

\section{Data analysis}

Statistical analysis was performed using SPSS 17.0 software (SPSS Inc., Chicago, IL, USA). Differences in total ACE-T scores, age, and education were analyzed using one-way analysis of variance (ANOVA). Pairwise comparisons were performed using Bonferroni's test. A receiver operating characteristic (ROC) curve analysis was used as a measure of the accuracy of the ACE-T. The optimal cutoff for the best sensitivity and specificity were determined. The convergent valid- ity of the ACE-T with TMMSE and MoCA scales were measured using Pearson's correlation. Inter-rater reliability was also evaluated by Pearson's correlation. The internal consistency reliability within the ACE-T was assessed using Cronbach's alpha. p-values of less than 0.05 were considered to indicate statistical significance.

\section{RESULTS}

Table 1 shows the demographic characteristics and ACE-T scores for the NC, MCI, and dementia groups. The dementia group consists of Alzheimer's disease $(n=21)$, mixed type $(n=4)$, and vascular dementia $(n=5)$. Post-hoc analyses of total ACE-T scores demonstrated that there were significant differences among the three groups.

\section{Distinction between cognitively healthy elderly and MCI patients}

The NC was compared with the MCI group. The results of the ROC analyses demonstrated that the AUC was 0.98. The optimal cut-off scores to distinguish the NC from MCI was $75 / 76$, giving a sensitivity of 0.90 and specificity of 0.96 .

\section{Distinction between non-dementia and dementia patients}

In this analysis, NC and patients with MCI were categorized in the "non-dementia group" for comparison against the dementia group, yielding an AUC value of 0.99. At the optimal cutoff score of 61/62, the ACE-T displayed excellent sensitivity (1.0) and specificity (0.97) to differentiate dementia from the non-dementia population.

\section{Convergent validity}

The total score of the ACE-T correlated significantly with the total MMSE and MoCA scores, with Pearson correlations of $0.83(\mathrm{p}<0.001)$ and $0.85(\mathrm{p}<0.001)$, respectively.

\section{Reliability}

Inter-rater reliability of the ACE-T was evaluated with a Pearson's correlation of $1.0(n=20)$. In terms of internal consistency, the Cronbach's alpha for the ACE-T was 0.93 .

Table 1. Demographic characteristics and total score of the Thai version of the Addenbrooke's Cognitive Examination

\begin{tabular}{lcccccc}
\hline & NC & MCI & Dementia & NC vs MCI & NC vs dementia & MCI vs dementia \\
\hline Number of participants & 48 & 29 & 30 & & & \\
Age, years: mean (SD) & $65.6(6.3)$ & $70.7(7.4)$ & $76.9(7.4)$ & $*$ & $* *$ & $*$ \\
Education, years: mean (SD) & $10.5(5.2)$ & $8.6(5.5)$ & $7.7(4.2)$ & NS & NS & NS \\
ACE-T: mean (SD) & $86.1(6.8)$ & $67.8(7.4)$ & $43.5(11.1)$ & $* * *$ & $* * *$ & $* * *$ \\
\hline
\end{tabular}

${ }^{*} \mathrm{p}<0.05,{ }^{* *} \mathrm{p}<0.01,{ }^{* * *} \mathrm{p}<0.001$. NS: non significant, ACE-T: the Thai version of the Addenbrooke's Cognitive Examination, MCI: mild cognitive impairment, NC: normal controls 


\section{Correlation with education}

ACE-T scores were significantly correlated with years of education in the NC $(r=0.65, \mathrm{p}<0.001)$ and $\mathrm{MCI}$ groups $(\mathrm{r}=$ $0.46, p<0.05)$, but not in the dementia group $(r=0.33, p>0.05)$.

\section{Time requirement of the ACE-T}

The average time required for the $\mathrm{NC}, \mathrm{MCI}$, and dementia groups to complete the ACE-T was 20, 27, and 31 minutes, respectively.

\section{DISCUSSION}

This study demonstrated that the Thai version of the ACE (ACE-T) is a valid and reliable cognitive assessment tool in the detection of MCI and early dementia in the Thai population.

The ACE-T exhibits good sensitivity and specificity in detecting MCI, and excellent sensitivity and specificity for dementia. These diagnosis accuracies are comparable with the original ACE III study, ${ }^{1}$ which has a sensitivity of 1.0 and specificity of 0.96 in dementia detection. In concurrence with the findings of the Thai version of the MMSE study, our study showed that the ACE-T was superior to the TMMSE. The TMMSE exhibited lower sensitivities in detecting dementia; in people with more than 6 years of education, sensitivity is 0.92 and specificity is 0.93 , and for people with less than 6 years of education, sensitivity is 0.57 and specificity is 0.94 .

In the original ACE, the mean total score for the $\mathrm{NC}$ was 95.4, and the recommended cut-off score for diagnosing dementia was $88,{ }^{1}$ while that of the Thai ACE was 86.1 , and the cut-off score was 61 . This difference may be because some questions in the ACE are based on Western culture and difficult for Thai people; for example, naming the harp, barrel, or the American president may be more difficult for Thai citizens. Supporting this idea, studies of the original ACE and that of and other developed countries had a higher mean score than in Asian countries, although levels of education are comparable. For example, the German ACE exhibited a mean score of 90.4 and cut-off score of $82,{ }^{4}$ higher than the Japanese ACE, which exhibited a mean score of 88.1 and cut-off score of $74 .^{6}$

The ACE-T required around 20 minutes to administer for the NC, and 27-31 minutes for the MCI and dementia groups. Because administering the MMSE usually takes approximately 8-13 minutes, ${ }^{10}$ the ACE-T may not be suitable for busy general practice clinics or for screening large populations. Nevertheless, the ACE measures a broad range of cognitive functions and comprises of questions of variable difficulty, which could better serve a specialist clinic like a memory or psychiatric clinic.

Our study had some limitations. Because most of our dementia patients had received a diagnosis of Alzheimer's disease, the generalizability with other types of dementia, like frontotemporal dementia, was limited. Prior studies demonstrated that the ACE could be applied to differentiate between frontotemporal dementia and Alzheimer's disease. ${ }^{1}$ There was also a difference in average age among groups; therefore, further studies should investigate age effects. Additionally, because the participants in this study were native Thai speakers with at least four years of education, the optimal cutoff point found in this study may not apply to people who use a Thai dialect as a first language or who have less education. In addition, test-retest reliability was not evaluated in this study.

\section{Acknowledgments}

The authors thank Prof. John Hodges for permission to translate the ACE III. This work was supported by a research grant from Thammasat University; fiscal year 2015 (Grant number 2558).

\section{REFERENCES}

1. Hsieh S, Schubert S, Hoon C, Mioshi E, Hodges JR. Validation of the Addenbrooke's Cognitive Examination III in frontotemporal dementia and Alzheimer's disease. Dement Geriatr Cogn Disord 2013;36:242-250.

2. Mioshi E, Dawson K, Mitchell J, Arnold R, Hodges JR. The Addenbrooke's Cognitive Examination Revised (ACE-R): a brief cognitive test battery for dementia screening. Int J Geriatr Psychiatry 2006;21:10781085.

3. Boustani M, Peterson B, Hanson L, Harris R, Lohr KN. Screening for dementia in primary care: a summary of the evidence for the US. Preventive Services Task Force. Ann Intern Med 2003;138:927-937.

4. Alexopoulos P, Ebert A, Richter-Schmidinger T, Schöll E, Natale B, Aguilar CA, et al. Validation of the German revised Addenbrooke's cognitive examination for detecting mild cognitive impairment, mild dementia in Alzheimer's disease and frontotemporal lobar degeneration. Dement Geriatr Cogn Disord 2010;29:448-456.

5. Fang R, Wang G, Huang Y, Zhuang JP, Tang HD, Wang Y, et al. Validation of the Chinese version of Addenbrooke's cognitive examination-revised for screening mild Alzheimer's disease and mild cognitive impairment. Dement Geriatr Cogn Disord 2014;37:223-231.

6. Yoshida H, Terada S, Honda H, Ata T, Takeda N, Kishimoto Y, et al. Validation of Addenbrooke's cognitive examination for detecting early dementia in a Japanese population. Psychiatry Res 2011;185:211-214.

7. American Psychiatric Association. Diagnostic and Statistical Manual of Mental Disorders, 5th Ed. Washington DC: American Psychiatric Association; 2013.

8. Institute of Geriatric Medicine. Thai Version of the Mini-Mental State Examination (MMSE-Thai 2002). Bangkok: Department of Medical Services; 2008.

9. Tangwongchai S, Phanasathit M, Charernboon T, Akkayagorn L, Hemrungrojn S, Phanthumchinda K, et al. The Validity of Thai version of The Montreal Cognitive Assessment (MoCA-T). Dement Neuropsychol 2009;3:172.

10. Cullen B, O’Neill B, Evans JJ, Coen RF, Lawlor BA. A review of screening tests for cognitive impairment. J Neurol Neurosurg Psychiatry 2007; 78:790-799. 